\title{
Effect of the tunneled-cuffed central venous catheters on oxidative stress indices and inflammation in chronic hemodialysis patients
}

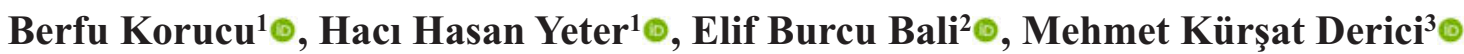 \\ ${ }^{1}$ Department of Nephrology, Gazi University School of Medicine, Ankara, Turkey \\ ${ }^{2}$ Department of Medical Services and Techniques, Gazi University Vocational School of Health Services, Ankara, Turkey \\ ${ }^{3}$ Department of Pharmacology, Kırlkkale University School of Medicine, Kırlkkale, Turkey
}

\section{ABSTRACT}

Objectives: The use of central venous catheters as hemodialysis (HD) vascular access is associated with worse morbidity and mortality in HD patients. This occasion is often attributed to comorbidities of the patients with central venous catheters. Studies reveal that a biofilm layer occurs on most of the tunneled-cuffed central venous catheters (TC-CVCs). This study aimed to determine the oxidative stress (OS) and systemic inflammation (SI) status in patients with TC-CVCs as HD vascular access without clinical signs and symptoms of infection.

Methods: The study is composed of eighty-five patients with a minimum HD vintage of one year. Patients with a history of infection or a cardiovascular event within six months, malignancy, systemic inflammatory diseases, or malnutrition were excluded. OS indices and SI markers were studied and compared in patients with arteriovenous fistula (AVF) and TC-CVCs.

Results: Mean native thiol/total thiol (NT/TT) ratio was significantly higher and mean disulphide/total thiol (DT/TT) ratio was significantly lower in AVF group comparing TC-CVC group $(0.46 \pm 0.17$ and $0.36 \pm 0.17$, $p=0.03$ for NT/TT; $0.27 \pm 0.08$ and $0.31 \pm 0.08, p=0.04$ for DS/TT; respectively). Mean OS index was significantly lower in the AVF group comparing TC-CVC group $(0.15 \pm 0.14$ and $0.24 \pm 0.23, p=0.04$; respectively]. Median hs-CRP levels and median IL-6 levels were significantly lower in AVF group comparing TC-CVC group (5.8 [min: 3.0-max: 82.5] mg/L and 9.7 [min: 3.0-max: 45.4] mg/L, $p=0.004$ for hs-CRP; 6.2 [min: 2.0-max:159.0] pg/mL and 12.2 [min: 2.6-max: 41.3) pg/mL, $p=0.01$ for IL-6; respectively).

Conclusions: TC-CVCs inversely affect OS and systemic inflammatory status in HD patients, presumably due to foreign body reactions and biofilm layers.

Keywords: Arteriovenous fistula, central venous catheters, hemodialysis vascular access, oxidative stress, systemic inflammation

$\mathrm{H}$ emodialysis (HD) is the most frequent renal replacement therapy (RRT) modality. HD requires a proper functioning vascular access (VA) such as arteriovenous fistula (AVF), arteriovenous graft (AVG), or central venous catheter (CVC). The use of CVCs is associated with worse morbidity and mortality in HD patients, which is usually attributed to patients' comorbidities, and these results are thought to be related to 
selection bias [1-4].

CVCs are widely produced from polyurethane, which is shown to induce mild foreign body reactions, although it is one of the most biocompatible substances [5]. CVCs interact with proteins and cells in the circulatory system and form an adherent biological material. When microorganisms settle on this material, it is called a biofilm layer [6]. Studies using quite sensitive techniques, including electron microscopy have reported the incidence of the biofilm layers at rates ranging from $36 \%$ to $100 \%$ in removed catheters of HD patients [7-9]. Thus, there is an increasing number of attempts to make polyurethane catheters more compatible and lead to less bacterial colonization [10].

Cardiovascular diseases (CVDs) are the most frequent cause of mortality in patients under RRT [11]. The two of the most critical factors for CVDs are oxidative stress (OS), and systemic inflammation (SI). The HD procedure and kidney disease itself are triggers of OS and SI $[12,13]$. On the other hand, in HD patients, smoking, serum uric acid levels, fluid overload, dialyzer type, dialysate purity, anemia, intravenous iron, and diabetes have also been associated with OS and SI [14]. However, this foreign substance in the vessel may be contributing to morbidity and mortality via triggering OS and SI as a potential biofilm carrier without any signs of infection.

This study aimed to determine the OS and SI status in patients with tunneled-cuffed CVCs (TC-CVCs) as HD VA without clinical signs and symptoms of infection comparing patients with AVF.

\section{METHODS}

\section{Participants and Study Design}

The study is composed of eighty-five patients with a minimum HD vintage of one-year. Informed consent was obtained from all patients. Patients with a history of infection or a cardiovascular event within six months were excluded. Patients with a history of malignancy, systemic inflammatory diseases, and malnutrition also excluded. Patients with AVGs, tunneled femoral or temporary catheters, patients treated with high-flux dialyzers or anticoagulated with citrate or low molecular weight heparin, and patients with catheters locked with antibiotics were also excluded for standardization (Fig. 1). Patients undergoing he- modialysis with TC-CVC were those with unfunctional AVF/AVG (68.2\%) and those who choose catheter use because of renal transplantation plan from a living donor in the near future $(31.8 \%)$.

All of the patients were under a standard HD procedure via AVF or polyurethane TC-CVC, thriceweekly (12 hours/week), using bicarbonate-containing dialysate and low-flux polysulfone membrane. All patients were anticoagulated with heparin. The blood flow rate ranged from 300 to $350 \mathrm{~mL} / \mathrm{min}$, and the dialysate flow rate was $500 \mathrm{~mL} / \mathrm{min}$. All tunneledcuffed CVCs locked with heparinized saline after HD.

The average of ultrafiltration rate within the last month, cumulative intravenous iron and erythropoiesis-stimulating agent (ESA) doses applied within the last six months were calculated. Doses of darbepoetin were converted to equivalent doses of epoetin for standardization [15]. Patients with $>100 \mathrm{ml} /$ day urine output were considered to have a residual renal function (RRF) [16].

\section{Blood Specimen Collection}

Blood specimens were collected at the initiation of a mid-week session. Specimens were allowed to clot at room temperature for 30 minutes. Clot removed by centrifuging samples at $3500 \mathrm{rpm}$ for 10 minutes. The serum immediately transferred into a polypropylene tube and stored at $-80 \mathrm{oC}$.

\section{Materials and Measurements}

The thiol measurements, OS indices, and inflammatory parameters were measured from freshly collected serum. Laboratory parameters (within three months for intact parathormone and two weeks for others) and demographic features were recorded from patients' files.

Single pool Kt/V calculated by Daugirdas' secondgeneration formula. Urea reduction ratio (URR) calculated by taking the difference between pre- and post-dialysis urea levels and divided by predialysis urea levels.

\section{Biochemical study}

Thiol/disulfide homeostasis measured using a novel automatic spectrophotometric method (Rel Assay Diagnostics, Turkey) [17]. Total oxidant status (TOS) and total antioxidant status (TAS) levels were measured using commercially available kits (Rel 


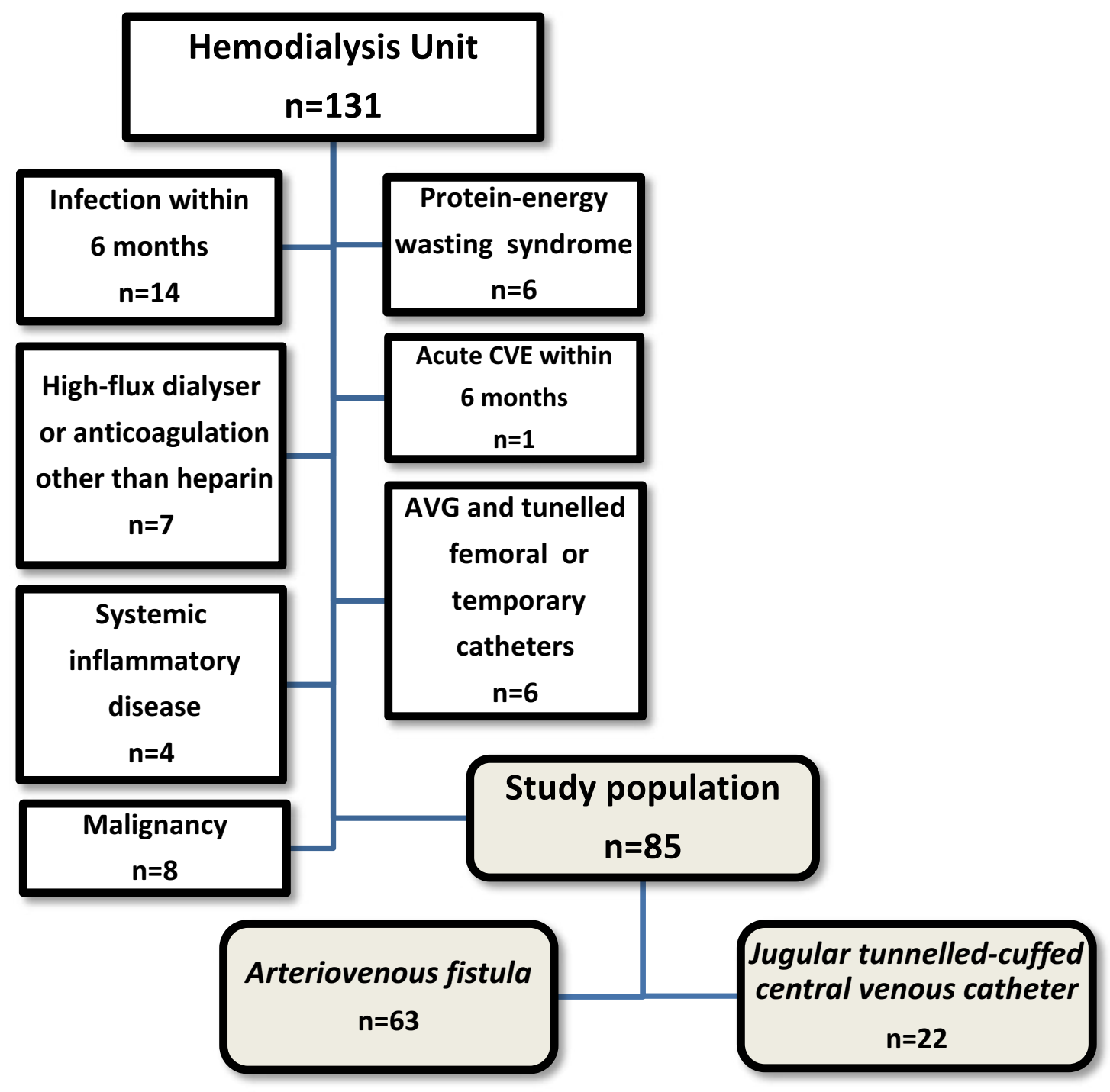

Fig. 1. Study design, study population, and groups. CVE = Cardiovascular event, AVG = Arteriovenous graft.

Assay Diagnostics, Turkey) [18]. The ratio of TOS to TAS accepted as the OS index (OSI). For calculation, the unit of TAS converted to $\mu \mathrm{mol} / \mathrm{L}$, and the OSI calculated according to the following formula: OSI (arbitrary unit $)=$ TOS $(\mu \mathrm{mol} \mathrm{H} 2 \mathrm{O} 2$ equivalent $/ \mathrm{L}) / \mathrm{TAS}$ ( $\mu$ mol Trolox equivalent/L). For regression analysis, cases with a TOS $>5 \mu \mathrm{mol} \mathrm{H} 2 \mathrm{O} 2$ equivalent/L were grouped as elevated score according to the manufacturer's recommendation.

Serum hs-CRP levels determined by a nephelometric technique using BNII/BN Pro-Spec (Siemens, Marburg, Germany); serum IL-6 levels determined by chemiluminescence immunoassay technique using Immulite 2000 (Siemens Diagnostics, Gwynedd, UK).

\section{Ethical statements}

The institute's committee (Kirıkkale University Clinical Research Ethics Committee) has approved the study protocol on human research (Decision No: 14/03, dated 27.6.2019).

\section{Statistical Analysis}

Data were expressed as mean \pm standard deviation or medians with ranges. For data normally distributed; Student's t-test is used for comparison between two groups. Inflammatory markers were non-normal distributed, and the Kruskal-Wallis test performed. A pvalue of less than 0.05 was considered statistically significant. All statistical analyses were performed using the Statistical Package for Social Science (SPSS, Chicago, IL, USA) for personal computers, version 21.0. 


\section{RESULTS}

\section{Baseline characteristics}

Demographic features, frequencies of comorbid diseases, frequency of smokers, laboratory parameters, HD adequacy indices, mean ultrafiltration rates, and cumulative doses of iron and ESAs were similar between groups. The frequency of patients with the RRF was higher in the TC-CVC group (Table 1).

\section{Oxidative stress measurements}

Mean TT and DS levels were significantly lower in the AVF group comparing the TC-CVC group (Fig. 2 ). The mean NT/TT ratio was significantly higher, and the mean DS/TT ratio was significantly lower in the AVF group comparing the TC-CVC group (Table 2, Fig. 3).

Mean TOS levels were significantly lower in the AVF group comparing the TC-CVC group. Mean TAS levels were similar between groups. Mean OSI was significantly lower in the AVF group comparing TCCVC group (Table 2, Figs. 3 and 4).

Table 1. Baseline characteristics and health parameters

\begin{tabular}{|c|c|c|c|}
\hline & $\begin{array}{c}\text { AVF } \\
(n=63)\end{array}$ & $\begin{array}{c}\text { TC-CVC } \\
(n=22)\end{array}$ & $p$ value \\
\hline Female/male & $22 / 41$ & $12 / 10$ & 0.07 \\
\hline Age (years) & $55.2 \pm 17.0$ & $63.9 \pm 18.7$ & 0.28 \\
\hline BMI $\left(\mathrm{kg} / \mathrm{m}^{2}\right)$ & $24.9 \pm 5.5$ & $24.9 \pm 4.8$ & 0.97 \\
\hline DM, n (\%) & $13(20.6)$ & $3(13.6)$ & 0.50 \\
\hline CAD, n (\%) & $17(26.9)$ & $7(31.8)$ & 0.61 \\
\hline $\mathrm{PAD} / \mathrm{CVD}, \mathrm{n}(\%)$ & $8(12.6)$ & $3(13.6)$ & 0.87 \\
\hline Smoking, n (\%) & $10(15.8)$ & $4(18.1)$ & 0.56 \\
\hline Urea nitrogen $(\mathrm{mg} / \mathrm{dL})$ & $62.8 \pm 14.8$ & $61.6 \pm 15.8$ & 0.59 \\
\hline Creatinine (mg/dL) & $8.4 \pm 1.9$ & $7.1 \pm 2.5$ & 0.15 \\
\hline Sodium (mEq/L) & $139.5 \pm 2.3$ & $138.8 \pm 2.0$ & 0.42 \\
\hline Potassium (mEq/L) & $4.9 \pm 0.7$ & $4.7 \pm 0.6$ & 0.39 \\
\hline Uric acid (mg/dL) & $5.3 \pm 0.9$ & $5.2 \pm 1.0$ & 0.80 \\
\hline Hemoglobin (g/dL) & $11.2 \pm 1.5$ & $10.5 \pm 1.6$ & 0.09 \\
\hline Albumin (g/dL) & $3.8 \pm 0.3$ & $3.6 \pm 0.4$ & 0.13 \\
\hline Calcium (mg/dL) & $8.8 \pm 0.8$ & $8.7 \pm 0.7$ & 0.85 \\
\hline Phosphorus (mg/dL) & $4.9 \pm 1.2$ & $4.7 \pm 1.3$ & 0.49 \\
\hline iPTH (pg/mL) & $452.7 \pm 422.9$ & $349.3 \pm 336.8$ & 0.31 \\
\hline $\mathrm{Kt} / \mathrm{V}$ & $1.6 \pm 0.2$ & $1.6 \pm 0.3$ & 0.85 \\
\hline URR (\%) & $73.7 \pm 6.8$ & $74.2 \pm 8.0$ & 0.80 \\
\hline HD vintage (months) & $76.0 \pm 67.1$ & $71.1 \pm 47.8$ & 0.61 \\
\hline Mean UF (mL) & $2400(500-3500)$ & $2300(1700-3000)$ & 0.08 \\
\hline RRF n (\%) & $17(26.9)$ & $9(40.9)$ & 0.05 \\
\hline Cum. IV Iron (mg) & $749.2 \pm 434.7$ & $713.6 \pm 425.7$ & 0.71 \\
\hline Cum. ESA (IU) & $60000(0-180800)$ & $64000(0-176000)$ & 0.82 \\
\hline
\end{tabular}

Data are shown as mean \pm standard deviation or $\mathrm{n}(\%)$ or median (minimum-maximum). AVF $=$ Arteriovenous fistula, TC-CVC $=$ Tunneled cuffed central venous catheter, BMI $=$ Body mass index, DM $=$ Diabetes mellitus, $\mathrm{CAD}=$ Coronary artery disease, $\mathrm{PAD} / \mathrm{CVD}=$ Peripheral artery disease/cerebrovascular disease, $\mathrm{iPTH}=$ intact Parathormone, $\mathrm{URR}=$ Urea reduction ratio, $\mathrm{HD}=$ Hemodialysis, $\mathrm{UF}=$ Ultrafiltration, $\mathrm{RRF}=$ Residual renal function, Cum. IV Iron $=$ Cumulative intravenous iron, Cum. ESA $=$ Cumulative erythropoiesis-stimulating agents 


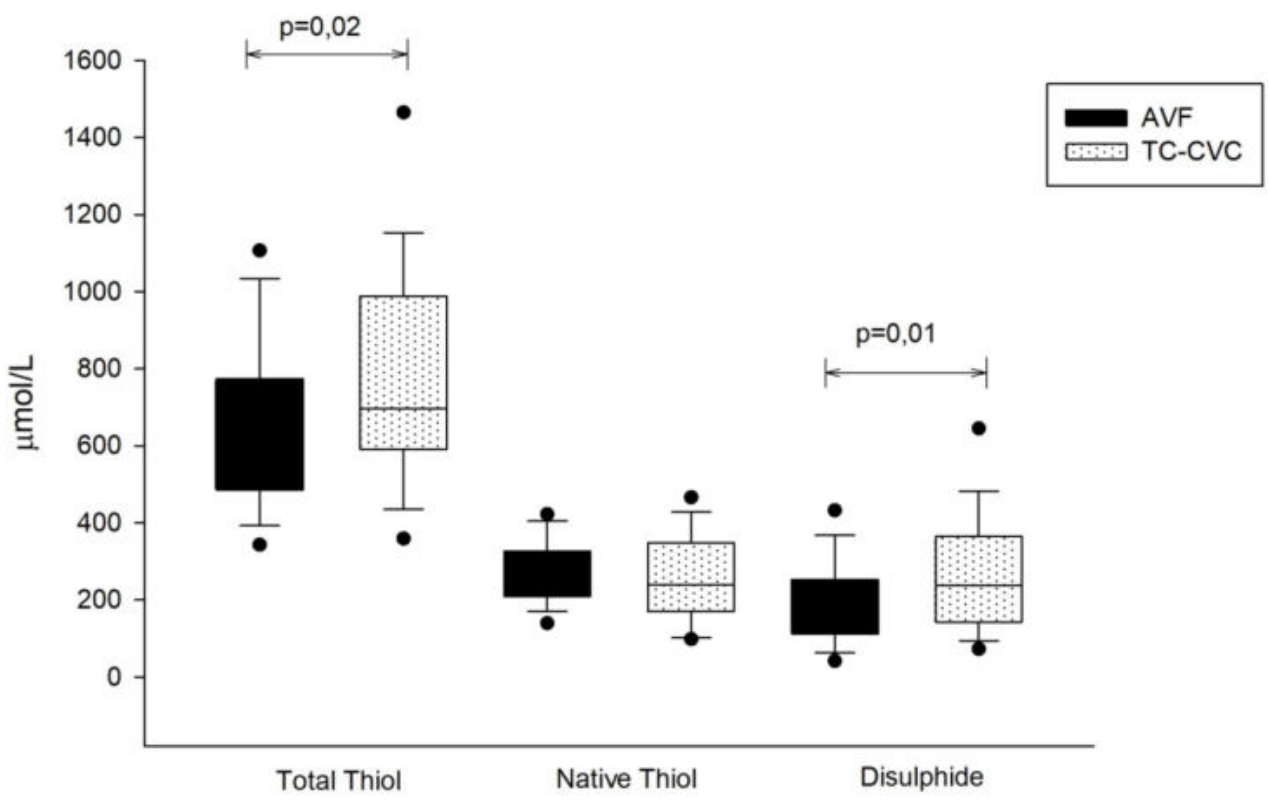

Fig. 2. Thiol measurements of the groups. AVF $=$ Arteriovenous fistula, $T C-C V C=$ Tunneled cuffed central venous catheter.

Table 2. Oxidative stress indices and inflammatory markers of the groups

\begin{tabular}{lccc}
\hline & $\begin{array}{c}\text { AVF } \\
(\mathbf{n}=\mathbf{6 3})\end{array}$ & $\begin{array}{c}\text { TC-CVC } \\
(\mathbf{n}=\mathbf{2 2})\end{array}$ & $\boldsymbol{p}$ value \\
\hline TT $(\boldsymbol{\mu m o l} / \mathbf{L})$ & $647 \pm 233.5$ & $788.2 \pm 285.1$ & $\mathbf{0 . 0 2}$ \\
NT $(\boldsymbol{\mu m o l} / \mathbf{L})$ & $272.2 \pm 86.6$ & $256.2 \pm 110.3$ & 0.49 \\
\hline DS $(\boldsymbol{\mu m o l} / \mathbf{L})$ & $187.5 \pm 112.9$ & $265.9 \pm 152.9$ & $\mathbf{0 . 0 1}$ \\
NT/TT & $0.46 \pm 0.17$ & $0.36 \pm 0.17$ & $\mathbf{0 . 0 3}$ \\
\hline DS/TT & $0.27 \pm 0.08$ & $0.31 \pm 0.08$ & $\mathbf{0 . 0 2}$ \\
TOS $(\boldsymbol{\mu m o l} / \mathbf{L})$ & $3.4 \pm 3.2$ & $5.5 \pm 5.7$ & $\mathbf{0 . 0 4}$ \\
\hline TAS $(\mathbf{m m o l} / \mathbf{L})$ & $2.3 \pm 0.2$ & $2.2 \pm 0.2$ & 0.29 \\
OSI $)$ & $0.15 \pm 0.14$ & $0.24 \pm 0.23$ & $\mathbf{0 . 0 4}$ \\
hs-CRP (mg/L) & $5.8(3.0-82.5)$ & $9.7(3.0-45.4)$ & $\mathbf{0 . 0 0 4}$ \\
\hline IL-6 (pg/mL) & $6.2(2.0-159.0)$ & $12.2(2.6-41.3)$ & $\mathbf{0 . 0 1}$ \\
\hline
\end{tabular}

Data are shown as mean \pm standard deviation or median (minimum-maximum). AVF $=$ Arteriovenous fistula, TC$\mathrm{CVC}=$ Tunneled cuffed central venous catheter, $\mathrm{TT}=$ Total thiol, NT $=$ Native thiol, $\mathrm{DS}=$ Disulphide, TOS $=$ Total oxidant status, TAS $=$ Total antioxidant status, OSI $=$ Oxidative stress index, hs-CRP $=$ high sensitive C-reactive protein, IL-6 = Interleukin-6.

\section{Inflammatory markers}

Median hs-CRP levels and median IL-6 levels were significantly lower in the AVF group comparing the TC-CVC group (Table 2, Fig. 4).

\section{Logistic regression analysis of risk factors for ele- vated total oxidative status}

When corrected for all possible risk factors, TC-
CVC significantly increases the risk of elevated TOS scores [OR: 6.90 (min: $1.09-\max : 43.6), p=0.04$ ] (Table 3).

\section{DISCUSSION}

CVCs are recommended to be the last choice for 


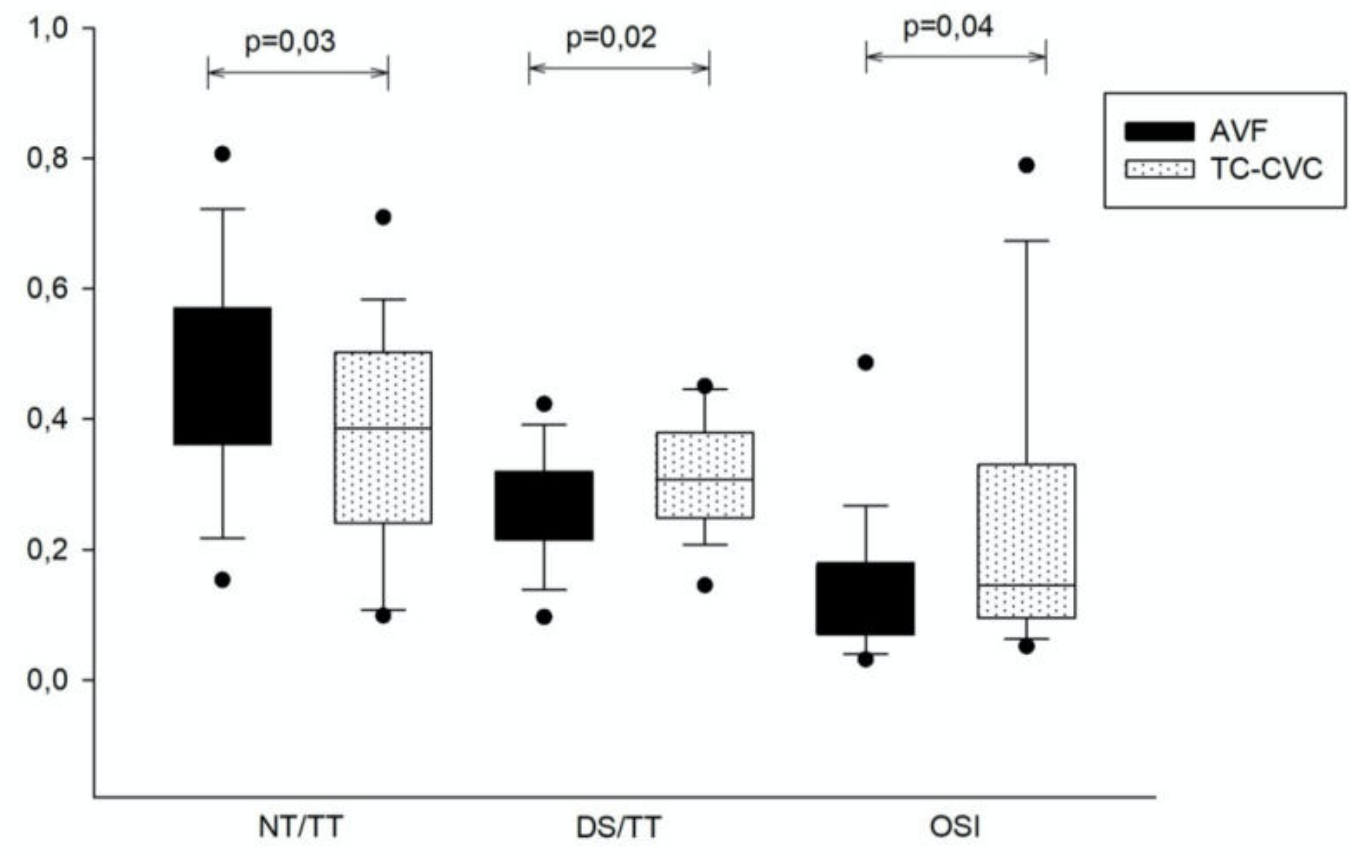

Fig. 3. Thiol balances of the groups. AVF = Arteriovenous fistula; TC-CVC $=$ Tunneled cuffed central venous catheter.

Table 3. Logistic regression analysis of risk factors for elevated total oxidative status

\begin{tabular}{lcc}
\hline & OR $(\mathbf{9 5 \%}$ CI) & p-value \\
\hline Age & $1.10(0.95-1.08)$ & 0.17 \\
BMI $\left(\mathrm{kg} / \mathrm{m}^{2}\right)$ & $1.78(0.59-1.20)$ & 0.93 \\
DM & $3.24(0.24-42.6)$ & 0.37 \\
Vascular disease* & $5.68(0.40-80.7)$ & 0.19 \\
Smoking & $6.16(0.44-85.8)$ & 0.17 \\
Cum. IV iron $(\mathrm{mg})$ & $0.99(0.99-1.00)$ & 0.07 \\
Mean UF $(\mathrm{mL})$ & $0.65(0.99-1.00)$ & 0.65 \\
hs-CRP $(\mathrm{mg} / \mathrm{L})$ & $0.34(0.96-1.11)$ & 0.34 \\
TC-CVC & $\mathbf{6 . 9 0 ( 1 . 0 9 - 4 3 . 6 )}$ & $\mathbf{0 . 0 4}$ \\
\hline BMI & IV &
\end{tabular}

$\mathrm{BMI}=$ Body mass index, DM $=$ Diabetes mellitus, Cum. IV Iron $=$ Cumulative intravenous iron, $\mathrm{UF}=$ Ultrafiltration, hs-CRP $=$ high sensitive $\mathrm{C}$-reactive protein, $\mathrm{TC}-\mathrm{CVC}=$ Tunneled cuffed central venous catheter

*Coronary artery disease, peripheral artery disease and/or cerebrovascular disease

HD VA by current guidelines. This approach is due to the observed higher mortality in patients with CVCs $[1-3,19,20]$. Most of these studies have not reported the causes of death. A recent well-designed study revealed a discordance in mortality rates and reported acute catheter complications [21].

Following the placement of the CVC, a layer of macromolecules, cells, and fibrinogen are rapidly deposited on the surface [6]. Microbial contamination of the layer can be as short as 24 hours. Intraluminal col- onization, in particular, is reported to be detected in $49-75 \%$ of patients with chronic HD catheters [9]. Bacteriae adhered to the biofilm produces an extracellular matrix that facilitates the adhesion of other pathogens and forms resistance to antibiotics and the host immune system [22]. The planktonic bacteriae can spread in circulation and cause bloodstream infections. However, there are emerging suspicions that this biofilm layer can also lead to OS and a silent SI [23]. $\mathrm{OS}$ is a risk factor for cardiovascular mortality 


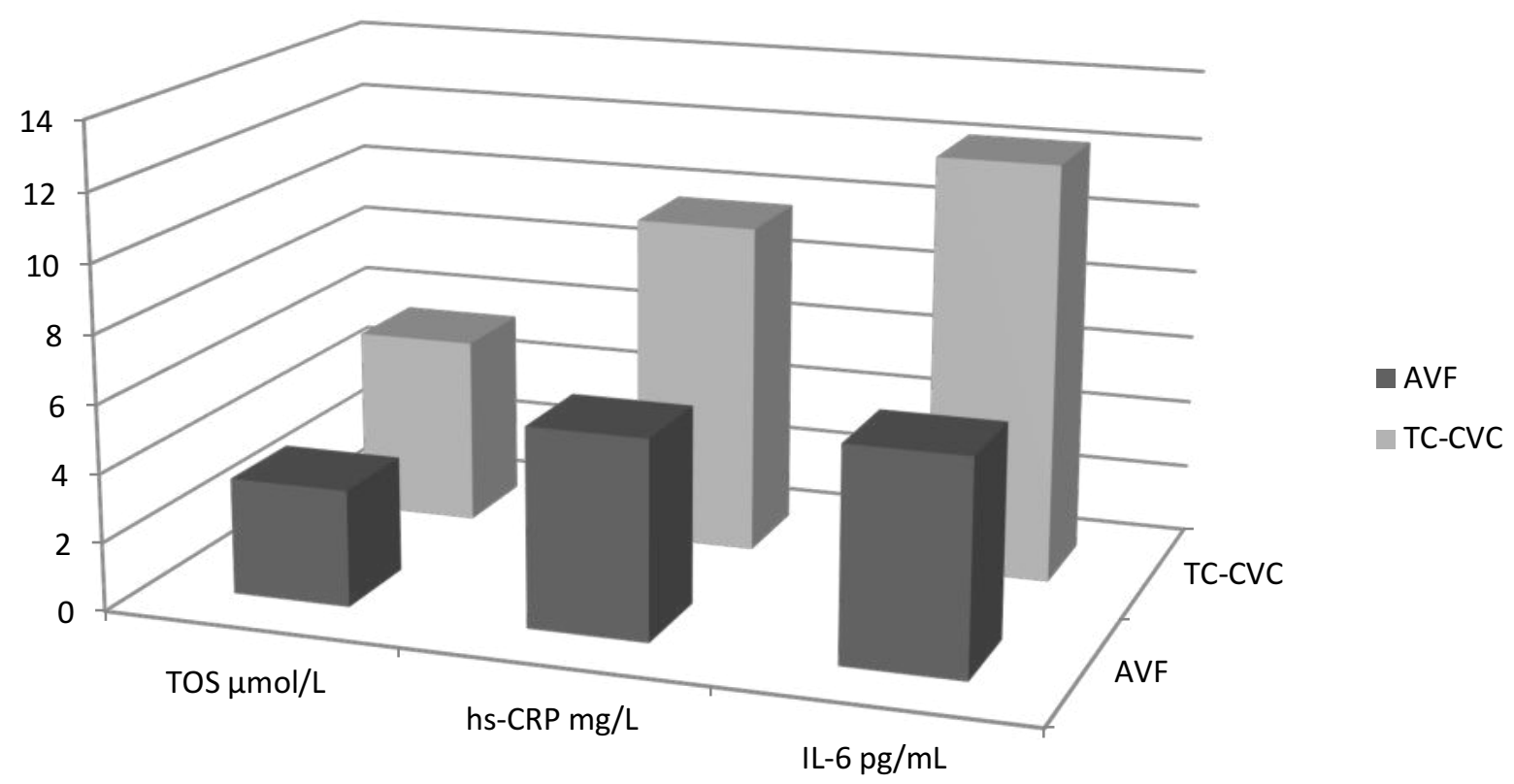

Fig. 4. Thiol balances of the groups. AVF = Arteriovenous fistula; TC-CVC $=$ Tunneled cuffed central venous catheter.

preferentially via oxidation of low-density lipoprotein in endothelium forming plaques [24]. SI is also a plaque trigger and a risk factor for CVDs [25]. The increased OS and SI have also been demonstrated in HD patients and associated with CVDs [26]. In our study, considering the proven OS factors in HD patients, we investigated the relationship between the presence of TC-CVC and the relationship between OS and SI using novel and sensitive markers.

Reactive oxygen species results in oxidation between two electrons or redox modification of radicalbased amino acid residues. In this redox reaction, the - $\mathrm{SH}$ groups of organosulfur compounds (thiols) such as cysteine oxidized and form a disulfide [27]. Thus, the dynamic thiol/disulfide homeostasis moves towards the disulfide form, which is the first sign of radical-mediated protein oxidation. This shift is the reflection of increased OS. Together with thiol-disulfide equilibrium, TAS and TOS measurements were made, and the OSI was calculated. Hs-CRP and IL-6 levels were used to determine SI.

The TC-CVC group had higher TT levels and DS levels, and more importantly, had lower NT/TT ratios that reflect superior antioxidant capability and higher DT/TT ratios that reflect oxidant stress. TOS levels and calculated OSI were also significantly higher in the TC-CVC group comparing the AVF group. On the other hand, the TC-CVC group had significantly higher hs-CRP and IL-6 levels.

The study revealed a clear difference between AVF and TC-CVC groups in terms of SI, consonant to the literature [28]. Furthermore, this is the first study to demonstrate the relationship between TC-CVC and OS with novel sensitive markers. All possible factors that may interact with these parameters such as, diabetes prevalence, vascular diseases, smoking, iron, and ESA therapies were similar between the groups. Also, TC-CVC was found to be an independent risk factor for elevated TOS. Moreover, patients who had an infectious disease and had any vascular event within the last six months were excluded from the study.

A meta-analysis composed of 62 studies reported that the proportion of access-related fatal infections in available studies was not precise, and in one study, only $23 \%$ of all infection-related hospitalizations were caused by access infection, suggesting that infections do not entirely explain the increased mortality associated with access types. In this meta-analysis, patients with CVCs had higher risks for all-cause mortality and cardiovascular events [29]. In a study of 4854 patients, AVF use 90 days after dialysis initiation was found to be associated with lower cardiovascular mortality compared with CVC use. The authors hypothesized that the biofilm in synthetic CVCs might increase the risk of CVD in HD patients via systemic inflammation [30]. 
Clinicians should bear in mind that OS and SI further increased by the use of TC-CVC may be a cause of worsening cardiovascular outcomes in HD patients. AVGs should be applied when AVF is not possible due to the patient's vascular structure. However, it should be remembered that peritoneal dialysis (PD) is an equivalent method applicable in almost all patients. PD should be introduced to every dialysis patient and encouraged to be used more frequently. For a small number of patients with no AVF or AVG chance and contraindications for PD, more biocompatible, less biofilm-producing CVCs with added antimicrobial properties may be used.

\section{CONCLUSION}

In HD patients, TC-CVCs inversely affect OS and SI status, presumably due to foreign body reactions and biofilm layers. However, VA through AVF is associated with a less oxidant state, and it is practically predictable that these more favorable effects will have ameliorating effects on adverse cardiovascular outcomes in these patients.

\section{Authors' Contribution}

Study Conception: BK; Study Design: BK, HHY, EBB; Supervision: MKD; Funding: BK; Materials: BK, HHY, EBB; Data Collection and/or Processing: BK, HHY, EBB; Statistical Analysis and/or Data Interpretation: BK, HHY, MKD; Literature Review: BK, HHY, MKD; Manuscript Preparation: BK and Critical Review: MKD.

\section{Conflict of interest}

The authors disclosed no conflict of interest during the preparation or publication of this manuscript.

\section{Financing}

The authors disclosed that they did not receive any grant during the conduction or writing of this study.

\section{REFERENCES}

1. Pastan S, Soucie JM, McClellan WM. Vascular access and increased risk of death among hemodialysis patients. Kidney Int 2002;62:620-6.
2. Astor BC, Eustace JA, Powe NR, Klag MJ, Fink NE, Coresh J; CHOICE Study. Type of vascular access and survival among incident hemodialysis patients: the Choices for Healthy Outcomes in Caring for ESRD (CHOICE) Study. J Am Soc Nephrol 2005;16:1449-55.

3. Polkinghorne KR, McDonald SP, Atkins RC, Kerr PG. Vascular access and all-cause mortality: a propensity score analysis. J Am Soc Nephrol 2004;15:477-86.

4. Brown RS, Patibandla BK, Goldfarb-Rumyantzev AS. The survival benefit of "Fistula First, Catheter Last" in hemodialysis is primarily due to patient factors. J Am Soc Nephrol 2017;28:64552.

5. Sheikh Z, Brooks P, Barzilay O, Fine N, Glogauer M. Macrophages, foreign body giant cells and their response to implantable biomaterials. Materials 2015;8:5671-701.

6. Murga R, Miller J, Donlan R. Biofilm formation by gram-negative bacteria on central venous catheter connectors: effect of conditioning films in a laboratory model. J Clin Microbiol 2001;39:2294-7.

7. Perez E, Williams M, Jacob JT, Reyes MD, Tejedor SC, Steinberg JP, et al. Microbial biofilms on needleless connectors for central venous catheters: comparison of standard and silvercoated devices collected from patients in an acute care hospital. J Clin Microbiol 2014;52:823-31.

8. Kanaa M, Wright M, Sandoe J. Examination of tunnelled haemodialysis catheters using scanning electron microscopy. Clin Microbiol Infect 2010;16:780-6.

9. Mermel LA. What is the evidence for intraluminal colonization of hemodialysis catheters? Kidney Int 2014;86:28-33.

10. Francois P, Vaudaux P, Nurdin N, Mathieu H, Descouts P, Lew DP. Physical and biological effects of a surface coating procedure on polyurethane catheters. Biomaterials 1996;17:667-78. 11. Fox CS, Matsushita K, Woodward M, Bilo HJ, Chalmers J, Heerspink HJL, et al. Associations of kidney disease measures with mortality and end-stage renal disease in individuals with and without diabetes: a meta-analysis. Lancet 2012;380:1662-73.

12. Cristol J, Canaud B, Rabesandratana H, Gaillard H, Serre A, Mion C. Enhancement of reactive oxygen species production and cell surface markers expression due to haemodialysis. Nephrol Dial Transplant 1994;9:389-94.

13. Modlinger PS, Wilcox CS, Aslam S. Nitric oxide, oxidative stress, and progression of chronic renal failure. Semin Nephrol 2004;24:354-65.

14. Liakopoulos V, Roumeliotis S, Gorny X, Dounousi E, Mertens PR. Oxidative stress in hemodialysis patients: a review of the literature. Oxid Med Cell Longev 2017;2017:3081856.

15. Scott SD. Dose conversion from recombinant human erythropoietin to darbepoetin alfa: recommendations from clinical studies. Pharmacotherapy 2002;22(9P2):160S-5S.

16. Shemin D, Bostom AG, Laliberty P, Dworkin LD. Residual renal function and mortality risk in hemodialysis patients. Am J Kidney Dis 2001;38:85-90.

17. Erel O, Neselioglu S. A novel and automated assay for thiol/disulphide homeostasis. Clin Biochem 2014;47:326-32.

18. Erel O. A new automated colorimetric method for measuring total oxidant status. Clin Biochem 2005;38:1103-11.

19. Moist LM, Trpeski L, Na Y, Lok CE. Increased hemodialysis 
catheter use in Canada and associated mortality risk: data from the Canadian Organ Replacement Registry 2001-2004. Clin J Am Soc Nephrol 2008;3:1726-32.

20. Dhingra RK, Young EW, Hulbert-Shearon T, Leavey SF, Port FK. Type of vascular access and mortality in US hemodialysis patients. Kidney Int 2001;60:1443-51.

21. Quinn RR, Oliver MJ, Devoe D, Poinen K, Kabani R, Kamar $\mathrm{F}$, et al. The effect of predialysis fistula attempt on risk of allcause and access-related death. J Am Soc Nephrol 2017;28:61320.

22. Flemming H-C, Wingender J, Szewzyk U, Steinberg P, Rice SA, Kjelleberg S. Biofilms: an emergent form of bacterial life. Nat Rev Microbiol 2016;14:563-75.

23. Cappelli G, Tetta C, Canaud B. Is biofilm a cause of silent chronic inflammation in haemodialysis patients? A fascinating working hypothesis. Nephrol Dial Transplant 2005;20:266-70. 24. Aviram M, Dornfeld L, Rosenblat M, Volkova N, Kaplan M, Coleman R, et al. Pomegranate juice consumption reduces oxidative stress, atherogenic modifications to LDL, and platelet aggregation: studies in humans and in atherosclerotic apolipoprotein E-deficient mice. Am J Clin Nutr 2000;71:106276.
25. van Leuven SI, Franssen R, Kastelein J, Levi M, Stroes ES, Tak PP. Systemic inflammation as a risk factor for atherothrombosis. Rheumatology 2007;47:3-7.

26. Cachofeiro V, Goicochea M, De Vinuesa SG, Oubiña P, Lahera V, Luño J. Oxidative stress and inflammation, a link between chronic kidney disease and cardiovascular disease. Kidney Int Suppl 2008;(111):S4-S9.

27. Cremers CM, Jakob U. Oxidant sensing by reversible disulfide bond formation. J Biol Chem 2013;288:26489-96.

28. Banerjee T, Kim SJ, Astor B, Shafi T, Coresh J, Powe NR. Vascular access type, inflammatory markers, and mortality in incident hemodialysis patients: the Choices for Healthy Outcomes in Caring for End-Stage Renal Disease (CHOICE) Study. Am J Kidney Dis 2014;64:954-61.

29. Ravani P, Palmer SC, Oliver MJ, Quinn RR, MacRae JM, Tai DJ, et al. Associations between hemodialysis access type and clinical outcomes: a systematic review. J Amn Soc Nephrol 2013;24:465-73.

30. Wasse H, Speckman RA, McClellan WM. Arteriovenous fistula use is associated with lower cardiovascular mortality compared with catheter use among ESRD patients. Semin Dial 2008;21:483-9. 